\title{
Isoperimetric inequalities of the fourth order Neumann eigenvalues
}

Yanlin Deng ${ }^{1}$ and Feng $\mathrm{Du}^{1,2^{*}}$ (D)

${ }^{\text {*Correspondence: }}$

defengdu123@163.com

'School of Mathematics and

Physics, Jingchu University of

Technology, Jingmen, 448000,

P.R. China

${ }^{2}$ Faculty of Mathematics and Statistics, Key Laboratory of Applied Mathematics of Hubei Province,

Hubei University, Wuhan, 430062,

P.R. China

\begin{abstract}
In this paper, we obtain some isoperimetric inequalities for the first $(n-1)$ eigenvalues of the fourth order Neumann Laplacian on bounded domains in an $n$-dimensional Euclidean space. Our result supports strongly the conjecture of Chasman.
\end{abstract}

MSC: $35 \mathrm{P} 15 ; 53 C 40 ; 58 C 40$

Keywords: Eigenvalues; Neumann problem; Isoperimetric inequality

\section{Introduction}

Letting $\Omega$ be a bounded domain with a smooth boundary $\partial \Omega$ in the Euclidean space $\mathbb{R}^{n}$, we consider the Neumann problem of the Laplacian $\Delta$ as follows:

$$
\begin{cases}\Delta u=\mu u, & \text { in } \Omega \\ \frac{\partial u}{\partial v}=0, & \text { on } \partial \Omega\end{cases}
$$

where $v$ is the outward unit normal to the boundary. It is well known that the free membrane problem (1.1) has a discrete spectrum consisting of a sequence

$$
0=\mu_{0}<\mu_{1} \leq \mu_{2} \leq \cdots \rightarrow+\infty \text {. }
$$

When $\Omega$ is a bounded domain in $\mathbb{R}^{2}$, Szegö [6] proved the following classical isoperimetric inequality:

$$
\mu_{1}(\Omega) \leq \mu_{1}\left(B_{\Omega}\right)
$$

where $B_{\Omega}$ is the ball of same volume as $\Omega$. Weinberger [11] generalized this result to $n$ dimensions. Ashbaugh and Benguria [2] extended the Szegö-Weinberger inequality (1.2) to the bounded domains in hyperbolic space and a hemisphere. On the other hand, Ashbaugh and Benguria [1] conjectured that

$$
\sum_{i=1}^{n} \frac{1}{\mu_{i}(\Omega)} \geq \frac{n}{\mu_{1}\left(B_{\Omega}\right)}, \quad \text { with equality if and only if } \Omega \text { is a ball, }
$$

(c) The Author(s) 2020. This article is licensed under a Creative Commons Attribution 4.0 International License, which permits use, sharing, adaptation, distribution and reproduction in any medium or format, as long as you give appropriate credit to the original author(s) and the source, provide a link to the Creative Commons licence, and indicate if changes were made. The images or other third party material in this article are included in the article's Creative Commons licence, unless indicated otherwise in a credit line to the material. If material is not included in the article's Creative Commons licence and your intended use is not permitted by statutory regulation or exceeds the permitted use, you will need to obtain permission directly from the copyright holder. To view a copy of this licence, visit http://creativecommons.org/licenses/by/4.0/. 
where $\mu_{i}(\Omega)$ is the $i$ th Neumann eigenvalue on $\Omega, \mu_{1}\left(B_{\Omega}\right)$ is the first nonzero Neumann eigenvalue on $B_{\Omega}$. In [10], Wang and Xia proved an isoperimetric inequality for the sums of the reciprocals of the first $(n-1)$ nonzero eigenvalues of the Neumann Laplacian on bounded domains in $\mathbb{R}^{n}$ as follows:

$$
\sum_{i=1}^{n-1} \frac{1}{\mu_{i}(\Omega)} \geq \frac{n-1}{\mu_{1}\left(B_{\Omega}\right)}, \quad \text { with equality if and only if } \Omega \text { is a ball, }
$$

which means the Ashbaugh-Benguria's conjecture is true for the first $(n-1)$ nonzero eigenvalues of the Neumann Laplacian on bounded domains in $\mathbb{R}^{n}$. So (1.4) supports the above conjectures of Ashbaugh and Benguria. On the other hand, Benguria, et al. [3] proved a result which is similar to (1.4) for the first $(n-1)$ nontrivial Neumann eigenvalues on domains in a hemisphere of $S^{n}$. Moreover, some works on eigenvalues are related to the spectra of matrix operators and can be seen in [7-9].

Let $\Delta$ and $\bar{\Delta}$ be the Laplace-Beltrami operators on $\Omega$ and $\partial \Omega$, respectively. Let $\nabla$ and $\bar{\nabla}$ be the gradient operators on $\Omega$ and $\partial \Omega$, respectively. Consider the following Neumann eigenvalue problem of the bi-harmonic operator:

$$
\begin{cases}\Delta^{2} u-\tau \Delta u=\Lambda u & \text { in } \Omega, \\ \frac{\partial^{2} u}{\partial v^{2}}=0, & \text { on } \partial \Omega, \\ \tau \frac{\partial u}{\partial v}-\operatorname{div}_{\partial \Omega}\left(\nabla^{2} u(v)\right)-\frac{\partial \Delta u}{\partial v}=0, & \text { on } \partial \Omega,\end{cases}
$$

where $\tau \geq 0$ and $\sigma$ are two constants, $\operatorname{div}_{\partial \Omega}$ denotes the tangential divergence operator on $\partial \Omega$, and $\nabla^{2} u$ is the Hessian of $u, v$ is the outward unit normal to the boundary. In this setting, problem (1.5) has a discrete spectrum, and all eigenvalues in the discrete spectrum can be listed nondecreasingly as follows:

$$
0=\Lambda_{0}<\Lambda_{1} \leq \Lambda_{2} \leq \cdots \uparrow+\infty
$$

By the Rayleigh-Ritz characterization, the $(k+1)$ th eigenvalue of $(1.5)$ can be given as follows (see, e.g., [5]):

$$
\Lambda_{k+1}=\inf _{u \in H^{2}(\Omega)}\left\{Q[u]=\frac{\int_{\Omega}\left[\left|\nabla^{2} u\right|^{2}+\tau|\nabla u|^{2}\right] d x}{\int_{\Omega} u^{2} d x} \mid \int_{\Omega} u u_{j}=0, j=1, \ldots, k\right\} .
$$

Letting $B_{\Omega}$ be the ball of same volume as $\Omega$, Chasman [5] proved the following isoperimetric inequality:

$$
\Lambda_{1}(\Omega) \leq \Lambda_{1}\left(B_{\Omega}\right), \quad \text { with equality if and only if } \Omega \text { is a ball. }
$$

Chasman [5] also conjectured that

$$
\sum_{i=1}^{n} \frac{1}{\Lambda_{i}(\Omega)} \geq \frac{n}{\Lambda_{1}\left(B_{\Omega}\right)}, \quad \text { with equality if and only if } \Omega \text { is a ball. }
$$

In this paper, we prove an isoperimetric inequality for the sums of the reciprocals of the first $(n-1)$ nonzero eigenvalues of the fourth Neumann Laplacian which supports the 
Chasman's conjecture, actually, we get

$$
\sum_{i=1}^{n-1} \frac{1}{\Lambda_{i}(\Omega)} \geq \frac{n-1}{\Lambda_{1}\left(B_{\Omega}\right)}, \quad \text { with equality if and only if } \Omega \text { is a ball. }
$$

In [4], Buoso et al. proved a quantitative isoperimetric inequality for the fundamental tone of problem (1.5) as follows:

$$
\Lambda_{1}(\Omega) \leq\left(1-\eta_{n, \tau,|\Omega|} A^{2}(\Omega)\right) \Lambda_{1}\left(B_{\Omega}\right)
$$

where $\eta_{n, \tau,|\Omega|}>0$, and $A(\Omega)$ is the so-called Fraenkel asymmetry of the domain $\Omega \in \mathbb{R}^{n}$, which is defined by:

$$
A(\Omega):=\inf \left\{\frac{\left|\Omega \Delta B_{\Omega}\right|}{|\Omega|}\right\}
$$

where $B_{\Omega}$ is the ball of same volume as $\Omega$ and $\Omega \Delta B_{\Omega}$ is the symmetric difference of $\Omega$ and $B_{\Omega}$. In what follows, we generalize (1.9) to the sum of the first $(n-1)$ eigenvalues, and we get

$$
\frac{1}{n-1} \sum_{i=1}^{n-1} \Lambda_{i}(\Omega) \leq\left(1-\eta_{n, \tau,|\Omega|} A^{2}(\Omega)\right) \Lambda_{1}\left(B_{\Omega}\right)
$$

\section{Preliminaries}

In this section, we recall some notations and results, more details can be seen in $[4,5]$.

Let $j_{1}, i_{1}$ be the ultraspherical and modified ultraspherical Bessel functions of the first kind and order 1 , respectively; $j_{1}, i_{1}$ can be expressed by the standard Bessel and modified Bessel functions of the first kind $J_{v}, I_{v}$ as follows:

$$
j_{1}(z)=z^{1-n / 2} J_{n / 2}(z), \quad i_{1}(z)=z^{1-n / 2} I_{n / 2}(z) .
$$

Let $B$ be the unit ball in $\mathbb{R}^{n}$ centered at the origin and $\omega_{n}$ be the Lebesgue measure $|B|$ of $B$, and let $\Lambda_{1}(B)$ be the first eigenvalue of problem (1.5) on unit ball $B$. For $\tau>0, a, b$ are positive constants satisfying the conditions $a^{2} b^{2}=\lambda_{1}(B)$ and $b^{2}-a^{2}=\tau$. Set

$$
R(r)=j_{1}(a r)+\gamma i_{1}(b r), \quad \gamma=\frac{-a^{2} j_{1}^{\prime \prime}(a r)}{b^{2} i_{1}^{\prime \prime}(b)}>0
$$

Then we define the function $\rho:[0,+\infty) \rightarrow[0,+\infty)$ as

$$
\rho(r)= \begin{cases}R(r), & r \in[0,1), \\ R(1)+(r-1) R^{\prime}(1), & r \in[1,+\infty) .\end{cases}
$$

Let $u_{i}: \mathbb{R}^{n} \rightarrow \mathbb{R}$ be defined by

$$
u_{i}(x):=\rho(|x|) \frac{x_{i}}{|x|}, \quad \text { for } i=1, \ldots, n
$$


The functions $\left.u_{i}\right|_{B}$ are, in fact, the eigenfunctions associated with the eigenvalues $\lambda_{1}(B)$ of problem (1.5) on unit ball $B$. We know that $\lambda_{1}(B)$ has multiplicity and $u_{i}$ satisfy

$$
\begin{aligned}
& \sum_{i=1}^{n}\left|u_{i}\right|^{2}=\rho^{2}(|x|), \\
& \sum_{i=1}^{n}\left|\nabla u_{i}\right|^{2}=\frac{n-1}{|x|^{2}} \rho(|x|)^{2}+\left(\rho^{\prime}(|x|)\right)^{2}, \\
& \sum_{i=1}^{n}\left|\nabla^{2} u_{i}\right|^{2}=\left(\rho^{\prime \prime}(|x|)\right)^{2}+\frac{3(n-1)}{|x|^{4}}\left[\rho(|x|)-|x| \rho^{\prime}(|x|)\right]^{2} .
\end{aligned}
$$

Define $N[\rho]=\sum_{i=1}^{n}\left(\left|\nabla^{2} u_{i}\right|^{2}+\tau\left|\nabla u_{i}\right|^{2}\right)$. Then $\rho$ and $N[\rho]$ satisfy the following properties which given in $[4,5]$.

Lemma 2.1 Function $\rho$ and $N[\rho]$ satisfy the following properties:

(1) $\rho^{\prime \prime}(r)<0$ for all $r \geq 0$, therefore $\rho^{\prime}$ is nonincreasing.

(2) $\rho(r)-r \rho^{\prime}(r) \geq 0$, with equality holding only for $r=0$.

(3) The function $\rho^{2}(r)$ is strictly increasing.

(4) The function $\rho^{2}(r) / r^{2}$ is decreasing.

(5) The function $3\left(\rho(r)-r \rho^{\prime}(r)\right)^{2} / r^{4}+\tau \rho^{2}(r) / r^{2}$ is decreasing.

(6) $N\left[\rho\left(r_{1}\right)\right]>N\left[\rho\left(r_{2}\right)\right]$ for any $r_{1} \in[0,1), r_{2} \in[1,+\infty)$.

(7) For all $r \geq 0$, we have

$$
N[\rho(r)]=\left(\rho^{\prime \prime}(r)\right)^{2}+\frac{3(n-1)\left(\rho(r)-r \rho^{\prime}(r)\right)^{2}}{r^{4}}+\tau(n-1) \frac{\rho^{2}(r)}{r^{2}}+\tau\left(\rho^{\prime}(r)\right)^{2} .
$$

(8) For all $r \geq 1, N[\rho(r)]$ is decreasing.

We introduce the notation of a partially monotonic function. A function $F$ is partially monotonic on $\Omega$ if it satisfies

$$
F(x)>F(y), \quad \text { for all } x \in \Omega \text { and } y \notin \Omega .
$$

It is seen that $N[\rho(r)]$ is a partially monotonic function from Lemma 2.1.

Lemma 2.2 For any radial function $F(r(x))$ that satisfies the partially monotonicity condition on $B_{\Omega}$,

$$
\int_{\Omega} F d x \leq \int_{B_{\Omega}} F d x
$$

with equality if and only if $\Omega=B_{\Omega}$. For any strictly increasing radial function $F(r(x))$,

$$
\int_{\Omega} F d x \geq \int_{B_{\Omega}} F d x
$$

with equality if and only if $\Omega=B_{\Omega}$. 
Lemma 2.3 For all $s>0$, we have

$$
\Lambda_{i}(\tau, \Omega)=s^{4} \Lambda_{i}\left(s^{-2} \tau, s \Omega\right), \quad i=1, \ldots, n,
$$

where $s \Omega=\left\{x \in \mathbb{R}^{n}: x / s \in \Omega\right\}$ for $s>0$.

Proof For any $u \in H^{2}(\Omega)$ with

$$
u \neq 0 \quad \text { and } \quad \int_{\Omega} u d x=\int_{\Omega} u u_{1} d x=\cdots=\int_{\Omega} u u_{i-1} d x=0, \quad i=1, \ldots, n,
$$

let $\tilde{u}(x)=u(x / s)$, then $\tilde{u}$ is a valid trial function on $s \Omega$ and so

$$
\begin{aligned}
Q_{s^{-2} \tau, s \Omega}[\tilde{u}] & =\frac{\int_{s \Omega}\left(\left|\nabla^{2} \tilde{u}\right|^{2}+s^{-2} \tau|\nabla \tilde{u}|^{2}\right) d x}{\int_{s \Omega} u^{2} d x} \\
& =\frac{\int_{s \Omega}\left(\left|s^{-2}\left(\nabla^{2} u\right)(x / s)\right|^{2}+s^{-2} \tau\left|s^{-1}(\nabla u)(x / s)\right|^{2}\right) d x}{\int_{s \Omega} u(x / s)^{2} d x} \\
& \left.=\frac{s^{-4+n} \int_{\Omega}\left(\left|\left(\nabla^{2} u\right)\right|^{2}+\tau|(\nabla u)|^{2}\right) d y}{s^{n} \int_{\Omega} u^{2} d y} \quad \text { (substituting } y=x / s\right) \\
& =s^{-4} Q_{\tau, \Omega}[u] .
\end{aligned}
$$

The lemma follows from (1.6).

\section{Proofs of the main results}

In this section, we give the proofs of the main results of this paper.

Theorem 3.1 Let $\Omega$ be a bounded domain in an n-dimensional Euclidean space $\mathbb{R}^{n}$ and let $B_{\Omega}$ be the ball of same volume as $\Omega$, then the first $(n-1)$ eigenvalues of $(1.5)$ in $\Omega$ satisfy

$$
\sum_{i=1}^{n-1} \frac{1}{\Lambda_{i}(\Omega)} \geq \frac{n-1}{\Lambda_{1}\left(B_{\Omega}\right)},
$$

with equality if and only if $\Omega$ is a ball.

Proof Assume that the volume of $\Omega$ is equal to that of the unit ball $B$. Letting $\varphi_{i}=\frac{\rho(r) x_{i}}{r}$, we know that

$$
\int_{\Omega} \varphi_{i}(r) d x=0, \quad \text { for } i=1, \ldots, n,
$$

which means $\varphi_{i}$ is perpendicular to $u_{0}=1 / \sqrt{|\Omega|}$, which is the first eigenfunction of (1.5). Letting $\left\{u_{j}\right\}_{j=0}^{\infty}$ be an orthonormal set of eigenfunctions of (1.5) on $\Omega$, next we will show that there exists new coordinate functions $\left\{x_{i}^{\prime}\right\}_{i=1}^{n}$ such that

$$
\int_{\Omega} \frac{\rho(r) x_{i}^{\prime}}{r} u_{j} d x=0
$$

for $j=1, \ldots, i-1$ and $i=2, \ldots, n$. To see this, we define an $n \times n$ matrix $A=\left(a_{i j}\right)$, where $a_{j i}=\int_{\Omega} \varphi_{i} u_{j} d x=\int_{\Omega} \frac{\rho(r)}{r} x_{i} u_{j} d x$, for $i, j=1,2, \ldots, n$. Using the orthogonalization of Gram 
and Schmidt (QR-factorization theorem), we know that there exist an upper-triangular matrix $T=\left(T_{j i}\right)$ and an orthogonal matrix $B=\left(b_{j i}\right)$ such that $T=U Q$, i.e.,

$$
T_{i j}=\sum_{k=1}^{n} b_{i k} a_{k j}=\int_{\Omega} \sum_{k=1}^{n} \frac{\rho(r)}{r} b_{i k} x_{k} u_{j} d x=0, \quad 1 \leq j<i \leq n .
$$

Letting $x_{i}^{\prime}=\sum_{k=1}^{n} b_{i k} x_{k}, i=1, \ldots, n$, we get (3.2). Since $B=\left(b_{j i}\right)$ is an orthogonal matrix, $\left\{x_{i}^{\prime}\right\}_{i=1}^{n}$ is also a set of coordinate functions. Therefore, denoting $x_{i}^{\prime}, i=1 \ldots, n$ still by $x_{i}$, $i=1 \ldots, n$, and $\varphi_{i}=\frac{\rho(r)}{r} x_{i}$, we have

$$
\varphi_{i} \neq 0 \quad \text { and } \quad \int_{\Omega} \varphi_{i} d x=\int_{\Omega} \varphi_{i} u_{1} d x=\cdots=\int_{\Omega} \varphi_{i} u_{i-1} d x=0, \quad i=1, \ldots, n .
$$

It follows from the Rayleigh-Ritz inequality that

$$
\Lambda_{i}(\Omega) \int_{\Omega} \varphi_{i}^{2} d x \leq \int_{\Omega}\left(\left|\nabla^{2} \varphi_{i}\right|^{2}+\tau\left|\nabla \varphi_{i}\right|^{2}\right) d x, \quad i=1, \ldots, n
$$

which implies that

$$
\int_{\Omega} \varphi_{i}^{2} d x \leq \frac{1}{\Lambda_{i}(\Omega)} \int_{\Omega}\left(\left|\nabla^{2} \varphi_{i}\right|^{2}+\tau\left|\nabla \varphi_{i}\right|^{2}\right) d x, \quad i=1, \ldots, n
$$

Summing over $i$ from 1 to $n$, we have

$$
\sum_{i=1}^{n} \int_{\Omega} \varphi_{i}^{2} d x \leq \sum_{i=1}^{n} \frac{1}{\Lambda_{i}(\Omega)} \int_{\Omega}\left(\left|\nabla^{2} \varphi_{i}\right|^{2}+\tau\left|\nabla \varphi_{i}\right|^{2}\right) d x
$$

Since $\sum_{i=1}^{n}\left|\nabla^{2} \varphi_{i}\right|^{2}=\left(\rho^{\prime \prime}\right)^{2}+\frac{3(n-1)}{r^{4}}\left(\rho-r \rho^{\prime}\right)^{2}$, for any point $p \in \Omega$, by a transformation of coordinates if necessary, we have $\left|\nabla^{2} \varphi_{i}\right|^{2} \leq \frac{\left(\rho^{\prime \prime}\right)^{2}}{n-1}+\frac{3}{r^{4}}\left(\rho-r \rho^{\prime}\right)^{2}, i=1, \ldots, n$. Then

$$
\begin{aligned}
& \sum_{i=1}^{n} \frac{1}{\Lambda_{i}(\Omega)}\left|\nabla^{2} \varphi_{i}\right|^{2} \\
& \quad=\sum_{i=1}^{n-1} \frac{1}{\Lambda_{i}(\Omega)}\left|\nabla^{2} \varphi_{i}\right|^{2}+\frac{1}{\Lambda_{n}(\Omega)}\left|\nabla^{2} \varphi_{i}\right|^{2} \\
& \quad=\sum_{i=1}^{n-1} \frac{1}{\Lambda_{i}(\Omega)}\left|\nabla^{2} \varphi_{i}\right|^{2}+\frac{1}{\Lambda_{n}(\Omega)}\left(\left(\rho^{\prime \prime}\right)^{2}+\frac{3(n-1)}{r^{4}}\left(\rho-r \rho^{\prime}\right)^{2}-\sum_{j=1}^{n-1}\left|\nabla^{2} \varphi_{j}\right|^{2}\right) \\
& \quad \leq \sum_{i=1}^{n-1} \frac{1}{\Lambda_{i}(\Omega)}\left|\nabla^{2} \varphi_{i}\right|^{2}+\sum_{j=1}^{n-1} \frac{1}{\Lambda_{j}(\Omega)}\left(\frac{\left(\rho^{\prime \prime}\right)^{2}+\frac{3(n-1)}{r^{4}}\left(\rho-r \rho^{\prime}\right)^{2}}{n-1}-\left|\nabla^{2} \varphi_{j}\right|^{2}\right) \\
& \quad=\frac{1}{n-1}\left(\left(\rho^{\prime \prime}\right)^{2}+\frac{3(n-1)}{r^{4}}\left(\rho-r \rho^{\prime}\right)^{2}\right) \sum_{i=1}^{n-1} \frac{1}{\Lambda_{i}(\Omega)} .
\end{aligned}
$$


Similarly, we have

$$
\begin{aligned}
\sum_{i=1}^{n} \frac{1}{\Lambda_{i}(\Omega)}\left|\nabla \varphi_{i}\right|^{2} & =\sum_{i=1}^{n-1} \frac{1}{\Lambda_{i}(\Omega)}\left|\nabla \varphi_{i}\right|^{2}+\frac{1}{\Lambda_{n}(\Omega)}\left|\nabla \varphi_{i}\right|^{2} \\
& =\sum_{i=1}^{n-1} \frac{1}{\Lambda_{i}(\Omega)}\left|\nabla \varphi_{i}\right|^{2}+\frac{1}{\Lambda_{n}(\Omega)}\left(\frac{n-1}{r^{2}} \rho^{2}+\left(\rho^{\prime}\right)^{2}-\sum_{j=1}^{n-1}\left|\nabla \varphi_{j}\right|^{2}\right) \\
& \leq \sum_{i=1}^{n-1} \frac{1}{\Lambda_{i}(\Omega)}\left|\nabla \varphi_{i}\right|^{2}+\sum_{j=1}^{n-1} \frac{1}{\Lambda_{j}(\Omega)}\left(\frac{\frac{n-1}{r^{2}} \rho^{2}+\left(\rho^{\prime}\right)^{2}}{n-1}-\left|\nabla \varphi_{j}\right|^{2}\right) \\
& =\frac{1}{n-1}\left(\frac{n-1}{r^{2}} \rho^{2}+\left(\rho^{\prime}\right)^{2}\right) \sum_{i=1}^{n-1} \frac{1}{\Lambda_{i}(\Omega)}
\end{aligned}
$$

On the other hand,

$$
\sum_{i=1}^{n}\left|\varphi_{i}\right|^{2}=\rho^{2}
$$

Substituting (3.6)-(3.8) into (3.5), we have

$$
\begin{aligned}
\frac{1}{n-1} \sum_{i=1}^{n-1} \frac{1}{\Lambda_{i}(\Omega)} & \geq \frac{\int_{\Omega} \rho^{2} d x}{\int_{\Omega}\left(\left(\rho^{\prime \prime}\right)^{2}+\frac{3(n-1)}{r^{4}}\left(\rho-r \rho^{\prime}\right)^{2}+\tau\left(\frac{n-1}{r^{2}} \rho^{2}+\left(\rho^{\prime}\right)^{2}\right)\right) d x} \\
& =\frac{\int_{\Omega} \rho^{2} d x}{\int_{\Omega} N[\rho] d x} \geq \frac{\int_{B_{\Omega}} \rho^{2} d x}{\int_{B_{\Omega}} N[\rho] d x}=\frac{1}{\Lambda_{1}\left(B_{\Omega}\right)},
\end{aligned}
$$

the last step is deduced by Lemma 2.2. If the equality holds, then equality holds in (3.9), which implies $\Omega$ must be a unit ball. By Lemma 2.3, for any domain $\Omega$ in $\mathbb{R}^{n}$, we get

$$
\frac{1}{n-1} \sum_{i=1}^{n-1} \frac{1}{\Lambda_{i}(\Omega)} \geq \frac{1}{\Lambda_{1}\left(B_{\Omega}\right)}
$$

This completes the proof of Theorem 3.1.

Theorem 3.2 Let $\Omega$ be a bounded domain in an n-dimensional Euclidean space $\mathbb{R}^{n}$ and let $B_{\Omega}$ be the ball of same volume as $\Omega$, then the first $(n-1)$ eigenvalues of $(1.5)$ in $\Omega$ satisfy

$$
\frac{1}{n-1} \sum_{i=1}^{n-1} \Lambda_{i}(\Omega) \leq\left(1-\eta_{n, \tau,|\Omega|} A^{2}(\Omega)\right) \Lambda_{1}\left(B_{\Omega}\right)
$$

Proof Case 1. $\Omega$ is a bounded domain in $\mathbb{R}^{n}$ of class $C^{1}$ with the same measure as the unit ball $B$. By a similar argument as in the proof of Theorem 3.1, we have

$$
\Lambda_{i}(\Omega) \int_{\Omega} \varphi_{i}^{2} d x \leq \int_{\Omega}\left(\left|\nabla^{2} \varphi_{i}\right|^{2}+\tau\left|\nabla \varphi_{i}\right|^{2}\right) d x, \quad i=1, \ldots, n .
$$


Summing over $i$ from 1 to $n$, we have

$$
\sum_{i=1}^{n} \Lambda_{i}(\Omega) \int_{\Omega} \varphi_{i}^{2} d x \leq \sum_{i=1}^{n} \int_{\Omega}\left(\left|\nabla^{2} \varphi_{i}\right|^{2}+\tau\left|\nabla \varphi_{i}\right|^{2}\right) d x=\int_{\Omega} N[\rho] d x .
$$

Since $\sum_{i=1}^{n} \varphi_{i}^{2}=\rho^{2}$, for any point $p \in \Omega$, by a transformation of coordinates if necessary, we have $\varphi_{i}^{2} \leq \frac{\rho^{2}}{n-1}, i=1, \ldots, n$. Then

$$
\begin{aligned}
\sum_{i=1}^{n} \Lambda_{i}(\Omega) \varphi_{i}^{2} & =\sum_{i=1}^{n-1} \Lambda_{i}(\Omega) \varphi_{i}^{2}+\Lambda_{n}(\Omega) \varphi_{n}^{2} \\
& =\sum_{i=1}^{n-1} \Lambda_{i}(\Omega) \varphi_{i}^{2}+\Lambda_{n}(\Omega)\left(\rho^{2}-\sum_{j=1}^{n-1} \varphi_{j}^{2}\right) \\
& \geq \sum_{i=1}^{n-1} \Lambda_{i}(\Omega) \varphi_{i}^{2}+\sum_{j=1}^{n-1} \Lambda_{j}\left(\frac{\rho^{2}}{n-1}-\varphi_{j}^{2}\right) \\
& =\sum_{i=1}^{n-1} \Lambda_{i} \frac{\rho^{2}}{n-1} .
\end{aligned}
$$

Substituting (3.13) into (3.14), we have

$$
\frac{1}{n-1} \sum_{i=1}^{n-1} \Lambda_{i}(\Omega) \leq \frac{\int_{\Omega} N[\rho] d x}{\int_{\Omega} \rho^{2} d x}
$$

On the other hand, we have

$$
\Lambda_{1}(B)=\frac{\int_{B} N[\rho] d x}{\int_{B} \rho^{2} d x}
$$

Combining (3.15) and (3.16), we have

$$
\Lambda_{1}(B) \int_{B} \rho^{2} d x-\frac{1}{n-1} \sum_{i=1}^{n-1} \Lambda_{i}(\Omega) \int_{\Omega} \rho^{2} d x \geq \int_{B} N[\rho] d x-\int_{\Omega} N[\rho] d x
$$

From equation (16) in [4], we know that

$$
\Lambda_{1}(B) \int_{B} \rho^{2} d x-\Lambda_{1}(\Omega) \int_{\Omega} \rho^{2} d x \leq C_{n, \tau}^{(1)}\left(\Lambda_{1}(B)-\Lambda_{1}(\Omega)\right),
$$

where $C_{n, \tau}^{(1)}=n \omega_{n} \int_{0}^{1} \rho^{2}(r) r^{n-1} d r$. Then we have

$$
\Lambda_{1}(B) \int_{B} \rho^{2} d x-\frac{1}{n-1} \sum_{i=1}^{n-1} \Lambda_{i}(\Omega) \int_{\Omega} \rho^{2} d x \leq C_{n, \tau}^{(1)}\left(\Lambda_{1}(B)-\frac{1}{n-1} \sum_{i=1}^{n-1} \Lambda_{i}(\Omega)\right) .
$$

From (15) and (20) in [4], we know that

$$
\Lambda_{1}(B) \int_{B} \rho^{2} d x-\Lambda_{1}(\Omega) \int_{\Omega} \rho^{2} d x \geq \int_{B / B_{1}} N(\rho) d x-\int_{B_{2} / B} N(\rho) d x,
$$


and

$$
\int_{B / B_{1}} N(\rho) d x-\int_{B_{2} / B} N(\rho) d x=C_{n, \tau}^{(2)} \alpha^{2}
$$

where $B_{1}$ and $B_{2}$ are two balls centered at the origin with radii $r_{1}, r_{2}$ such that $|\Omega \cap B|=$ $\left|B_{1}\right|=\omega_{n} r_{1}^{n}$ and $|\Omega / B|=\left|B_{2} / B\right|=\omega_{n}\left(r_{2}^{n}-1\right)$. Then we have

$$
\int_{B} N[\rho] d x-\int_{\Omega} N[\rho] d x \geq C_{n, \tau}^{(2)} \frac{|\Omega \Delta B|}{|\Omega|}
$$

where $C_{n, \tau}^{(2)}=n \omega_{n}\left((3+\tau)\left(R(1)-R^{\prime}(1)\right)^{2}+2 \tau R^{\prime}(1)\left(R(1)-R^{\prime}(1)\right)\right) c_{n}$.

Combining (3.18) and (3.19), we have

$$
\Lambda_{1}(B)-\frac{1}{n-1} \sum_{i=1}^{n-1} \Lambda_{i}(\Omega) \geq \frac{C_{n, \tau}^{(2)}}{C_{n, \tau}^{(1)}} A^{2}(\Omega),
$$

which implies that

$$
\frac{1}{n-1} \sum_{i=1}^{n-1} \Lambda_{i}(\Omega) \leq \Lambda_{1}(B)\left(1-\frac{C_{n, \tau}^{(2)}}{\Lambda_{1}(B) C_{n, \tau}^{(1)}} A^{2}(\Omega)\right)
$$

Case 2. $\Omega$ is the generic domain in $\mathbb{R}^{n}$ of class $C^{1}$. Since

$$
\Lambda_{i}(\tau, \Omega)=s^{4} \Lambda_{i}\left(s^{-2} \tau, s \Omega\right), \quad i=1, \ldots, n,
$$

for all $s>0$. Taking $s=\left(\omega_{n} /|\Omega|\right)^{\frac{1}{n}}$, for any domain $\Omega$ in $\mathbb{R}^{n}$ of class $C^{1}$, we infer from (3.21) that

$$
\begin{aligned}
\frac{1}{n-1} \sum_{i=1}^{n-1} \Lambda_{i}(\tau, \Omega) & =s^{4} \frac{1}{n-1} \sum_{i=1}^{n-1} \Lambda_{i}\left(s^{-2} \tau, s \Omega\right) \\
& \leq s^{4} \Lambda_{1}\left(s^{-2} \tau, B\right)\left(1-\frac{C_{n, s^{-2} \tau}^{(2)}}{\Lambda_{1}\left(s^{-2} \tau, B\right) C_{n, s^{-2} \tau}^{(1)}} A^{2}(s \Omega)\right) \\
& =\Lambda_{1}\left(s^{-2} \tau, B\right)\left(1-\frac{C_{n, s^{-2} \tau}^{(2)}}{\Lambda_{1}\left(s^{-2} \tau, B\right) C_{n, s^{-2} \tau}^{(1)}} A^{2}(\Omega)\right) .
\end{aligned}
$$

Setting $\eta_{n, \tau,|\Omega|}=\frac{C_{n, s^{-2} \tau}^{(2)}}{\Lambda_{1}\left(s^{-2} \tau, B\right) C_{n, s^{-2} \tau}^{(1)}}$, we have (1.10). This completes the proof of Theorem 3.2.

\section{Acknowledgements}

The authors would like to thank the referee for his or her careful reading and valuable comments such that the article appears in its present version.

\section{Funding}

This work is supported by NSF of Hubei Provincial Department of Education (Grant Nos. B2019211, D20184301, B2016261), Research Team Project of Jingchu University of Technology (Grant No. TD202006), Research Project of Jingchu University of Technology (Grant No. QDB201608) and Hubei Key Laboratory of Applied Mathematics (Hubei University). 
Availability of data and materials

Not applicable.

\section{Competing interests}

All authors declare that they have no competing interests.

\section{Authors' contributions}

All authors have equal contribution. All authors read and approved the final manuscript.

\section{Publisher's Note}

Springer Nature remains neutral with regard to jurisdictional claims in published maps and institutional affiliations.

Received: 28 May 2019 Accepted: 5 August 2020 Published online: 15 August 2020

\section{References}

1. Ashbaugh, M., Benguria, R.: Universal bounds for the low eigenvalues of Neumann Laplacians in N dimensions. SIAM J. Math. Anal. 24, 557-570 (1993)

2. Ashbaugh, M., Benguria, R.: Sharp upper bound to the first nonzero Neumann eigenvalue for bounded domains in spaces of constant curvature. J. Lond. Math. Soc. 52(2), 402-416 (1995)

3. Benguria, R., Brandolini, B., Chiacchio, F.: A sharp estimate for Neumann eigenvalues of the Laplace-Beltrami operator for domains in a hemisphere. arXiv preprint (2018). arXiv:1809.05695v1

4. Buoso, D., Chasman, L.M., Provenzano, L.: On the stability of some isoperimetric inequalities for the fundamental tones of free plates. J. Spectr. Theory 8(3), 843-869 (2018)

5. Chasman, L.M.: An isoperimetric inequality for fundamental tones of free plates. Commun. Math. Phys. 303(2), 421-449 (2011)

6. Szegö, G.: Inequalities for certain eigenvalues of a membrane of given area. J. Ration. Mech. Anal. 3, 343-356 (1954)

7. Tripathy, B.C., Paul, A.: The spectrum of the operator $D(r, 0, s, 0, t)$ over the sequence spaces $c_{0}$ and c. J. Math. 2013, Article ID 430965 (2013)

8. Tripathy, B.C., Paul, A.: The spectrum of the operator $D(r, 0,0, s)$ over the sequence space $c_{0}$ and $c$. Kyungpook Math. J. 53(2), 247-256 (2013)

9. Tripathy, B.C., Saikia, P.: On the spectrum of the Cesàro operator $C_{1}$ on $\overline{b v} \cap 1_{\infty}$. Math. Slovaca 63(3), 563-572 (2013)

10. Wang, Q., Xia, C.: On a conjecture of Ashbaugh and Benguria about lower eigenvalues of the Neumann Laplacian. arXiv preprint (2018). arXiv:1808.09520v3

11. Weinberger, H.F.: An isoperimetric inequality for the N-dimensional free membrane problem. J. Ration. Mech. Anal. 5 , 633-636 (1956)

\section{Submit your manuscript to a SpringerOpen ${ }^{\circ}$ journal and benefit from:}

- Convenient online submission

- Rigorous peer review

- Open access: articles freely available online

- High visibility within the field

- Retaining the copyright to your article 